\title{
Association of tumor morphology with long-term prognosis after liver resection for patients with a solitary huge hepatocellular carcinoma-a multicenter propensity score matching analysis
}

\author{
Xin-Fei Xu ${ }^{1 \#}$, Han $\mathrm{Wu}^{1 \#}$, Ju-Dong $\mathrm{Li}^{2 *}$, Lan-Qing Yao ${ }^{1 \#}$, Bin Huang ${ }^{3}$, Yong-Kang Diao ${ }^{4}$, Ting-Hao Chen ${ }^{5}$, \\ Wei-Min $\mathrm{Gu}^{6}$, Zhong Chen ${ }^{7}$, Jie $\mathrm{Li}^{8}$, Yao-Ming Zhang ${ }^{9}$, Hong Wang ${ }^{10}$, Ying-Jian Liang ${ }^{11}$, Ya-Hao Zhou ${ }^{12}$, \\ Chao Li ${ }^{1}$, Ming-Da Wang ${ }^{1}$, Cheng-Wu Zhang ${ }^{4}$, Timothy M. Pawlik ${ }^{13}$, Wan Yee Lau ${ }^{1,14}$, Feng Shen ${ }^{1}$, \\ Tian Yang ${ }^{1,4}$
}

${ }^{1}$ Department of Hepatobiliary Surgery, Eastern Hepatobiliary Surgery Hospital, Naval Medical University (Second Military Medical University), Shanghai, China; ${ }^{2}$ Department of General Surgery, Changzheng Hospital, Naval Medical University (Second Military Medical University), Shanghai, China; ${ }^{3}$ Department of Radiology, Eastern Hepatobiliary Surgery Hospital, Naval Medical University (Second Military Medical University), Shanghai, China; ${ }^{4}$ Department of General Surgery, Cancer Center, Division of Hepatobiliary and Pancreatic Surgery, Zhejiang Provincial People's Hospital, People's Hospital of Hangzhou Medical College, Hangzhou, China; ${ }^{5}$ Department of General Surgery, Ziyang First People's Hospital, Ziyang, China; ${ }^{6}$ The First Department of General Surgery, the Fourth Hospital of Harbin, Harbin, China; ${ }^{7}$ Department of Hepatobiliary Surgery, Affiliated Hospital of Nantong University, Nantong, China; ${ }^{8}$ Department of Hepatobiliary Surgery, Fuyang People's Hospital, Fuyang, China; ${ }^{9}$ The Second Department of Hepatobiliary Surgery, Meizhou People's Hospital, Meizhou, China; ${ }^{10}$ Department of General Surgery, Liuyang People's Hospital, Changsha, China; ${ }^{11}$ Department of Hepatobiliary Surgery, the First Affiliated Hospital of Harbin Medical University, Harbin, China; ${ }^{12}$ Department of Hepatobiliary Surgery, Pu'er People's Hospital, Pu'er, China; ${ }^{13}$ Department of Surgery, Ohio State University, Wexner Medical Center, Columbus, OH, USA; ${ }^{14}$ Faculty of Medicine, the Chinese University of Hong Kong, Hong Kong, China

Contributions: (I) Conception and design: XF Xu, H Wu, JD Li, LQ Yao, F Shen, T Yang; (II) Administrative support: XF Xu, H Wu, B Huang, H Wang, TH Chen, WM Gu, Z Chen, J Li, YM Zhang, CW Zhang, YJ Liang, YH Zhou, F Shen, T Yang; (III) Provision of study materials or patients: JD Li, H Wang, TH Chen, WM Gu, Z Chen, J Li, YM Zhang, CW Zhang, YH Zhou, T Yang; (IV) Collection and assembly of data: XF Xu, H Wu, JD Li, LQ Yao, YK Diao, H Wang, TH Chen, WM Gu, Z Chen, J Li, YM Zhang, CW Zhang, YJ Liang, YH Zhou, C Li, MD Wang, F Shen, T Yang; (V) Data analysis and interpretation: XF Xu, H Wu, JD Li, T Yang; (VI) Manuscript writing: All authors; (VII) Final approval of manuscript: All authors.

\#These authors contributed equally to this work.

Correspondence to: Prof. Tian Yang, MD; Prof. Feng Shen, MD, PhD. Department of Hepatobiliary Surgery, Eastern Hepatobiliary Surgery Hospital, Naval Medical University (Second Military Medical University), Shanghai, China. Email: yangtianehbh@smmu.edu.cn; fengshensmmu@gmail.com.

Background: A solitary hepatocellular carcinoma (HCC) without macrovascular invasion and distant metastasis, regardless of tumor size, is currently classified as early-stage disease by the latest Barcelona Clinic Liver Cancer (BCLC) staging system. While the preferred treatment is surgical resection, the association of tumor morphology with long-term survival outcomes after liver resection for a solitary huge HCC of $\geq 10 \mathrm{~cm}$ has not been defined.

Methods: Patients who underwent curative liver resection for a solitary huge HCC were identified from a multicenter database. Preoperative imaging findings were used to define spherical- or ellipsoidal-shaped lesions with smooth edges as balloon-shaped HCCs (BS-HCCs); out-of-shape lesions or lesions of any shape with matt edges were defined as non-balloon-shaped HCCs (NBS-HCCs). The two groups of patients with BS-HCCs and NBS-HCCs were matched in a 1:1 ratio using propensity score matching (PSM). Clinicopathologic characteristics, long-term overall survival (OS) and recurrence-free survival (RFS) were assessed.

Results: Among patients with a solitary huge HCC, 74 pairs of patients with BS-HCC and NBS-HCC were matched. Tumor pathological features including proportions of microvascular invasion, satellite nodules, and incomplete tumor encapsulation in the BS-HCC group were lower than the NBS-HCC group. At a median follow-up of 50.7 months, median OS and RFS of all patients with a solitary huge HCC after 
PSM were 27.8 and 10.1 months, respectively. The BS-HCC group had better median OS and RFS than the NBS-HCC group (31.9 vs. 21.0 months, $\mathrm{P}=0.01$; and 19.7 vs. 6.4 months, $\mathrm{P}=0.015$ ). Multivariate analyses identified BS-HCC as independently associated with better OS (HR 0.592, P=0.009) and RFS (HR 0.633, $\mathrm{P}=0.013)$.

Conclusions: For a solitary huge HCC, preoperative imaging on tumor morphology was associated with prognosis following resection. In particular, patients with BS-HCCs had better long-term survival following liver resection versus patients with large NBS-HCCs.

Keywords: Hepatocellular carcinoma (HCC); survival; recurrence; tumor morphology; hepatectomy

Submitted Oct 13, 2021. Accepted for publication Dec 23, 2021.

doi: $10.21037 / \mathrm{hbsn}-21-423$

View this article at: https://dx.doi.org/10.21037/hbsn-21-423

\section{Introduction}

Hepatocellular carcinoma (HCC) is the most common primary liver cancer with an estimated incidence of $>1$ million cases by 2025 (1). Surgery, in the form of liver resection or liver transplantation, remains the mainstay of treatment aimed at cure for patients with resectable diseases (2). The Barcelona Clinic Liver Cancer (BCLC) classification has been endorsed as the optimal staging system and treatment algorithm for HCC by the European Association for the Study of Liver Disease (EASL) and the American Association for the Study of Liver Disease (AASLD) $(3,4)$. Since first being proposed, the BCLC classification has been updated several times with the most notable update being staging of large solitary $\mathrm{HCC}>5 \mathrm{~cm}$. In the latest BCLC staging, solitary HCC, without vascular invasion and distant metastasis, regardless of tumor size is classified as early-staged HCC (BCLC stage 0/A). In turn, the recommended treatment is liver resection for patients having preserved liver functions (5). However, surgical resection for a solitary large $(>5 \mathrm{~cm})$ or huge $(>10 \mathrm{~cm})$ HCC remains a challenge even for experienced hepatic surgeons, with an increased risk of massive intraoperative hemorrhage and fatal postoperative complications (6-9). Good surgical decision-makings require comprehensive evaluations to balance expected survival benefits with potential surgical difficulty and risks (10-12). Therefore, good understanding of the clinicopathological characteristics and prognostic factors of patients being considered for liver resection of a solitary huge HCC, even those considered as early-staged HCC by the BCLC staging system, is important.

Pathological features relating to tumor biology and invasiveness associated with long-term oncologic prognosis have been identified in previous studies on liver resection for a solitary large or huge HCC (13-15). These features include tumor differentiation $(16,17)$, tumor encapsulation $(18,19)$, microvascular invasion $(14,20)$, and satellite nodules (20). Data on morphologic shape are scarce. Of note, most HCCs start to grow in the early stages in a spherical or ellipsoidal shape. As tumor size increases, more aggressive tumor features can manifest with loss of smooth tumor edges, breakthrough of the tumor envelope, loss in original spherical or ellipsoidal shapes, presence of satellites or multiple nodules around the primary tumors, micro- and macrovascular invasion, and distant metastases $(21,22)$. However, one specific type of solitary huge HCC that exhibits low invasive and metastatic potentials, and may have more favorable outcomes after curative resection has been described (Figure 1). In particular, a solitary huge HCC, despite its size of $>5 \mathrm{~cm}$ for a solitary large HCC and $>10 \mathrm{~cm}$ for a solitary huge HCC, that retains its spherical or ellipsoidal shape, smooth tumor edge, absence of local protrusion, and intact capsule or pseudocapsule has been suggested to have a better prognosis. This type of HCC is named "balloon-shaped" HCC based on its shape.

Although solitary large or huge HCCs have been reported to have specific molecular characteristics $(14,23,24)$, to our knowledge, no study has focused on the prognostic significance of tumor morphology among patients with solitary huge HCC after curative liver resection. In the current study, data on the clinicopathologic characteristics, preoperative computed tomography (CT) and/or magnetic resonance imaging (MRI) features, and perioperative and postoperative survival outcomes from a multicenter cohort of patients with solitary huge HCCs without macrovascular invasion and distant metastases were retrospectively analyzed. Using propensity score matching 

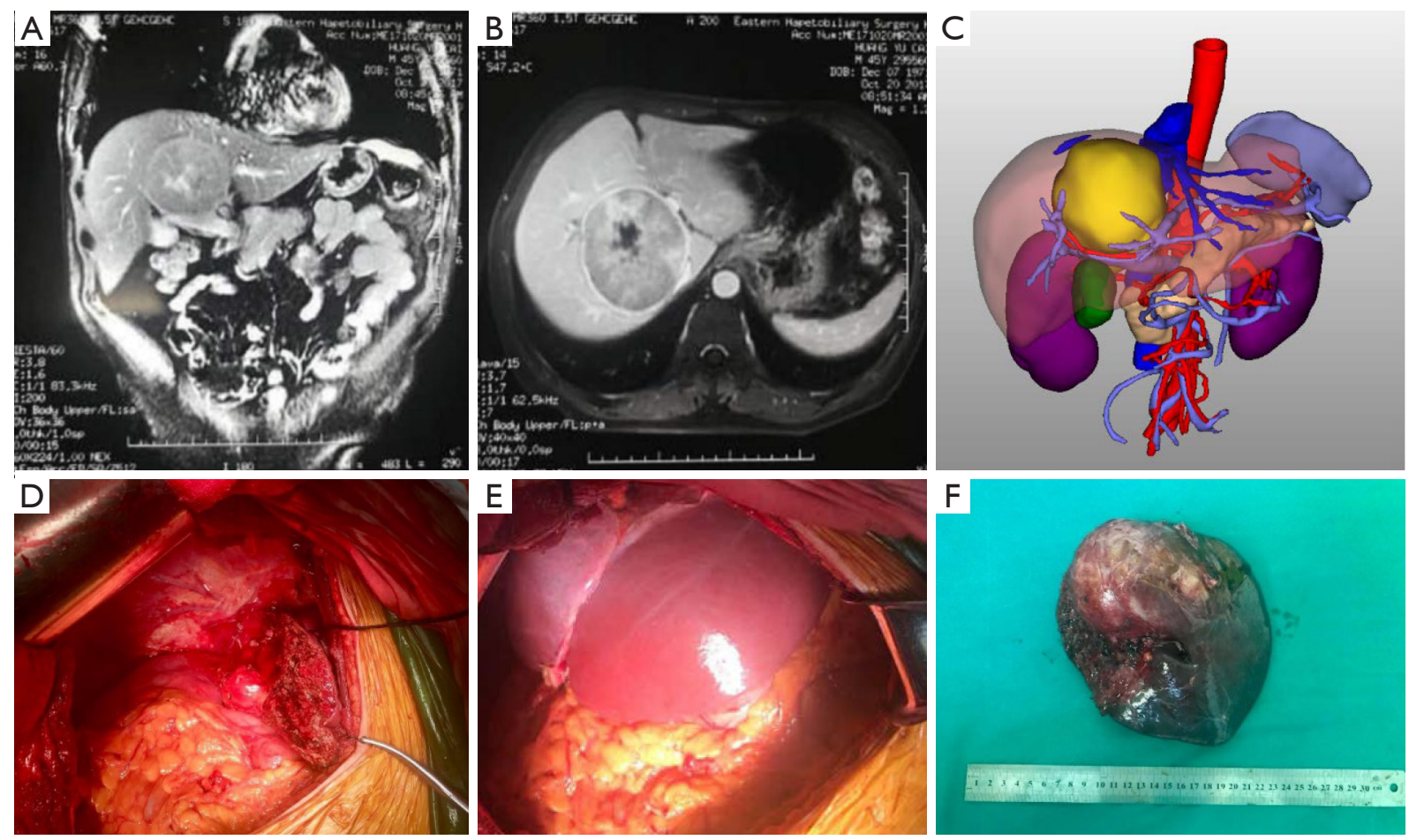

Figure 1 A representative set of magnetic resonance imaging (MRI), three-dimensional imaging, and operative photographs of a 45-year-old male with a solitary huge balloon-shaped hepatocellular carcinoma (BS-HCC) located between the left and right hemilivers. MRI (A,B) and three-dimensional imaging (C) show a solitary huge lesion located in segments 4, 5, and 8 (largest tumor size: $11.0 \mathrm{~cm}$ ). The preoperative alpha-fetoprotein (AFP) was $48.9 \mathrm{ng} / \mathrm{mL}$. This patient underwent curative extended right hepatectomy (D-F) on November 02, 2017, and was still alive and recurrence-free at the last follow-up on July 20, 2021.

(PSM), long-term survival and recurrence outcomes were compared between patients with balloon shaped-HCCs (BS-HCC) versus non-balloon-shaped HCCs (NBS-HCC) on survival outcomes after liver resection. In particular, we sought to define whether tumor morphology could be used to stratify patients with solitary huge HCCs over $10 \mathrm{~cm}$ in diameter relative to prognosis following resection. We present the following article in accordance with the STROBE reporting checklist (available at https://hbsn. amegroups.com/article/view/10.21037/hbsn-21-423/rc).

\section{Methods}

\section{Patients}

Using a multicenter database from 11 Chinese hospitals [Eastern Hepatobiliary Surgery Hospital (EHBH) of Shanghai, Changzheng Hospital of Shanghai, Liuyang People's Hospital, Ziyang First People's Hospital, Fourth Hospital of Harbin, First Affiliated Hospital of Nantong
University, Fuyang People's Hospital, Meizhou People's Hospital, Zhejiang Provincial People's Hospital, First Affiliated Hospital of Harbin Medical University, and Pu'er People's Hospital], patients who underwent curativeintent liver resection for newly diagnosed HCC from June 2007 to August 2020 were retrospectively reviewed. Approval was obtained from the Institutional Review Boards of these hospitals. The inclusion criteria were patients with: (I) a solitary HCC with a maximum diameter of $10 \mathrm{~cm}$ or more; (II) absence of macrovascular invasion and distant metastasis; (III) curative liver resection with complete removal of all microscopic and macroscopic tumors (R0 resection); (IV) adequate preoperative contrast-enhanced CT or MRI images, clinicopathological variables and follow-up data. The exclusion criteria were patients with: (I) age younger than 18 years; (II) multiple HCCs; (III) tumors with a maximum diameter $<10 \mathrm{~cm}$; (IV) preoperative anti-HCC treatment; (V) palliative liver resection, including microscopically positive (R1 
resection) or grossly positive (R2 resection) resection margins; (VI) unavailable data on preoperative CT/MRI or essential prognostic variables; (VII) loss to follow-up within 6 months after surgery. Data were collected in both a prospective and retrospective fashion depending on the data field. The study was performed in accordance with the Declaration of Helsinki and the Ethical Guidelines for Clinical Studies for the enrolled centers. Informed consent was waived by the Institutional Review Board of EHBH (No. EHBHKY2019-K-005). Consent for the relevant procedures and the use of data for research purposes were obtained from all the patients before treatment.

\section{Identifications of imaging features between BS-HCC and NBS-HCC}

The digital data on all the preoperative CT and/or MRI images carried out within 1 month before surgery of all the enrolled patients from the participating hospitals other than EHBH were sent to EHBH. The images were independently reviewed by one radiologist $(\mathrm{HB})$ and two surgeons (XXF and YLQ) who were blinded to patient clinicopathological information. Lesions were subsequently categorized as BS-HCC or NBS-HCC. Any discrepancies in classification were settled through discussion until a consensus was reached. According to preoperative imaging findings, a solitary huge BS-HCC had all the following features: (I) a spherical- or ellipsoidal-shaped lesion larger than $10 \mathrm{~cm}$; (II) smooth tumor edges without any local protrusion; and (III) a low- or high-density peripheral rim around the lesion. In contrast, out-of-shape lesions or lesions of any shape with matt edges on imaging were classified as NBS-HCCs.

\section{Clinicopathological variables and perioperative outcomes}

The medical records were retrospectively reviewed for clinicopathological variables. Preoperative clinical characteristics included age, sex, American Society of Anesthesiologists (ASA) score, hepatitis B virus infection, liver fibrosis, cirrhosis, Child-Pugh grading, preoperative platelets count, and preoperative alpha-fetoprotein (AFP) level. Preoperative imaging, data on tumor size, tumor location (involving one or both hemi-livers), tumor morphology (balloon type or non-balloon type), and tumor growing mode (pedunculated or not) were recorded. Perioperative outcomes included intraoperative blood loss, intraoperative blood transfusion, extent of hepatectomy (minor or major), type of hepatectomy (anatomical or non-anatomical), and postoperative 30-day mortality and morbidity. Major hepatectomy was defined as resection of three or more Couinaud liver segments. Anatomical hepatectomies were defined by the Brisbane 2000 nomenclature of liver anatomy (25), while non-anatomical hepatectomies included wedge resection or limited resection.

Postoperative pathological features collected relative to the liver and tumor included cirrhosis, microvascular invasion, satellite nodules, tumor encapsulation (no/ incomplete or complete), tumor differentiation (poor or well/moderate), and resection margin $(<1$ or $\geq 1 \mathrm{~cm})$. Tumor encapsulation was defined as the presence of a fibrous sheath around the tumor on gross inspection (18). Tumor differentiation was identified using the Edmondson-Steiner histopathological grading system (26). Pedunculated HCC reflected a special growth pattern of HCC with more than $50 \%$ of tumor volume protruding outside of liver parenchyma.

\section{Study endpoints}

Given that the study focused on the prognostic role of tumor morphology among patients with a solitary huge HCC who underwent curative liver resection, the primary endpoints were overall survival (OS) and recurrence-free survival (RFS); secondary endpoints included incidence of death and recurrence on follow-up. Tumor recurrence was clinically suspected with progressive elevation of serum AFP levels and ultrasonographic detection of a new hepatic lesion. The diagnosis of a recurrence was made when dynamic CT scan or MRI demonstrated contrast enhancement in the arterial phase and wash-out in the venous phase, or when hepatic angiography disclosed a high tumor vascularity. Patients developing recurrence were treated with re-resection, local ablation, liver transplantation, transcatheter arterial chemoembolization, radiotherapy, targeted therapy, or supportive care, depending on the patterns of recurrence, liver functional reserve, and patient general conditions. Re-resection, liver transplantation, and local ablation were defined as potentially curative treatments, while other treatments were deemed as noncurative treatments. OS was calculated from the date of liver resection to either the date of death or the date of the last follow-up, while RFS was calculated from the date of liver resection to the date of diagnosis of first recurrence, or the date of death or the last follow-up. 


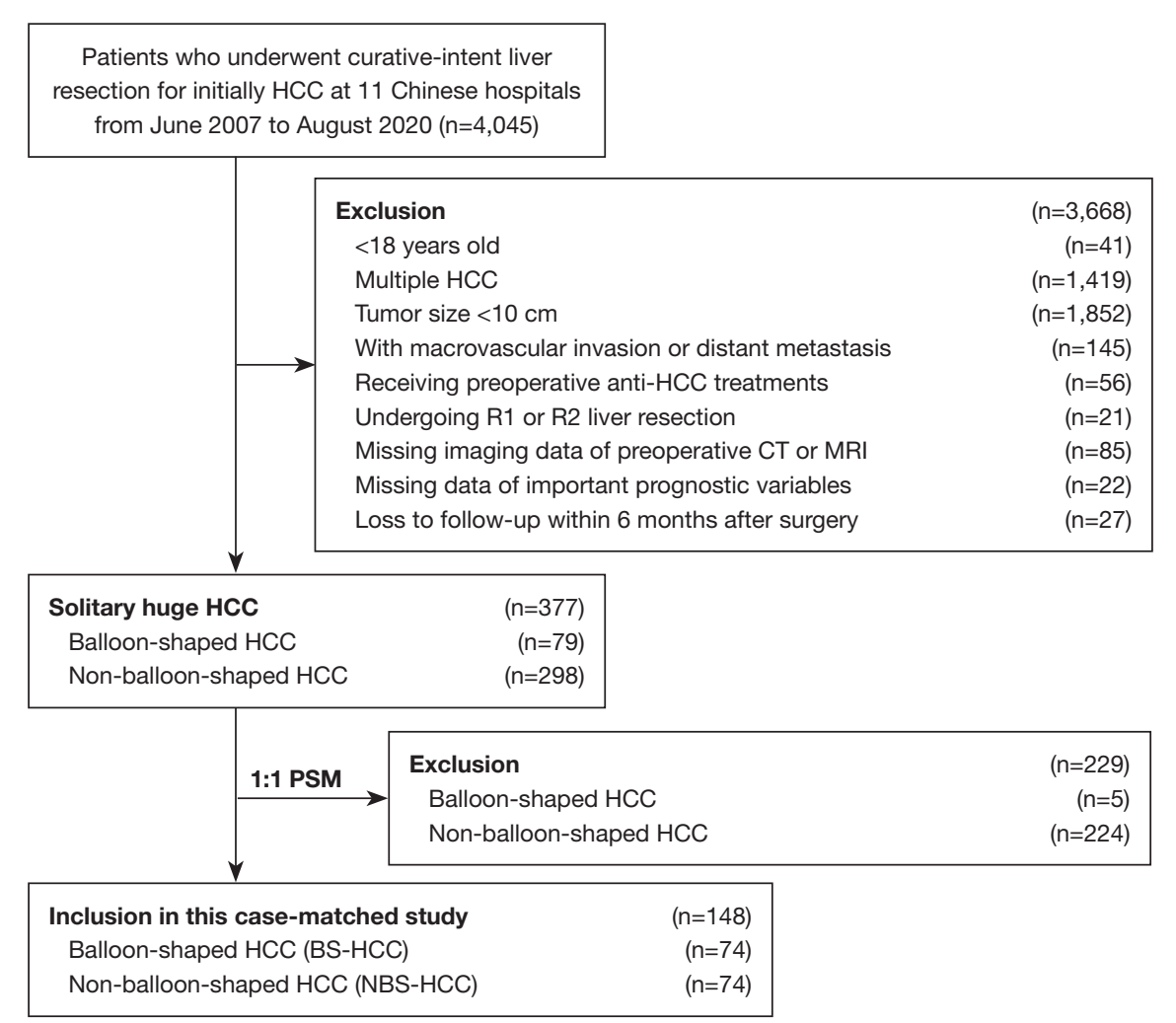

Figure 2 Flow chart of the study. HCC, hepatocellular carcinoma; PSM, propensity score matching.

\section{Statistical analysis}

Clinicopathological characteristics were summarized using frequency and percentage for categorical variables and mean \pm standard deviation (SD) or median (range) for continuous variables. The $\chi^{2}$ test or Fisher's exact test was used to compare categorical covariates, while continuous covariates were compared using the independent-samples $t$-test or Mann-Whitney U test between the PSM patients with BS-HCC and NBS-HCC. OS and RFS were calculated by the Kaplan-Meier methods and compared by the log-rank test. The univariate and multivariate Cox proportional hazard regression model was used to identify independent prognostic factors of OS and RFS. Variables with a $\mathrm{P}<0.10$ on univariate analysis were subjected to the multivariate Cox-regression model using a forward stepwise variable selection. Statistical analyses were performed using the SPSS software version 25.0 (SPSS, Chicago, IL, USA). A two-tailed P value of $<0.05$ was considered statistically significant for all the tests.

\section{Results}

Among patients who underwent resection for a solitary huge HCC, an initial analysis of variance and $\chi^{2}$ test for baseline demographics and preoperative imaging characteristics of patients demonstrated differences in certain variables, including Child-Pugh grading, tumor size, and uni- or bilateral hemi-liver location among patients with BS-HCC versus NBS-HCC. Using propensity scores to adjust for these 4 variables, a 1:1 PSM was conducted to create matched study cohorts of BS-HCC (n=74) and NBSHCC ( $\mathrm{n}=74)$ patients (Figure 2). The typical CT or MRI imaging findings of these 148 patients are displayed in the Figures S1,S2.

\section{Clinicopathological variables and perioperative outcomes}

Comparison of clinicopathologic characteristics and perioperative outcomes between the BS-HCC and NBSHCC groups are noted in Table 1. In the overall cohort, there were $122(82.4 \%)$ men and $26(17.6 \%)$ women with a mean age \pm SD of $51.0 \pm 12.4$ years. The mean \pm SD tumor diameter was $12.1 \pm 1.9 \mathrm{~cm}$. There were $48(32.4 \%)$ patients who had an HCC located between the left and right hemi-livers (bilateral hemi-liver location); 43 (29.1\%) 
Table 1 Clinicopathologic characteristics and perioperative outcomes after liver resection for patients with a solitary huge hepatocellular carcinoma

\begin{tabular}{|c|c|c|c|c|}
\hline Variables & Total $(n=148)$ & BS-HCC $(n=74)$ & NBS-HCC $(n=74)$ & $P$ \\
\hline Age, years* & $51.0 \pm 12.4$ & $52.0 \pm 12.6$ & $50.0 \pm 12.3$ & 0.339 \\
\hline Male sex & $122(82.4)$ & $59(79.7)$ & $63(85.1)$ & 0.388 \\
\hline ASA score $>2$ & $16(10.8)$ & $9(12.2)$ & $7(9.5)$ & 0.597 \\
\hline Fibrosis & $136(91.9)$ & $67(90.5)$ & 69 (93.2) & 0.547 \\
\hline Cirrhosis & $93(62.8)$ & $46(62.2)$ & $47(63.5)$ & 0.865 \\
\hline Child-Pugh grade B & $16(10.8)$ & $8(10.8)$ & $8(10.8)$ & 1.000 \\
\hline Preoperative platelets count, $\times 10^{9} / \mathrm{L}^{*}$ & $187 \pm 80$ & $192 \pm 85$ & $183 \pm 74$ & 0.472 \\
\hline \multicolumn{5}{|l|}{ Preoperative imaging characteristics } \\
\hline Bilobular location & $48(32.4)$ & $26(35.1)$ & $22(29.7)$ & 0.482 \\
\hline Tumor size, $\mathrm{cm}^{\star}$ & $12.1 \pm 1.9$ & $12.0 \pm 1.9$ & $12.3 \pm 1.9$ & 0.343 \\
\hline Pedunculated growing & $43(29.1)$ & $23(31.1)$ & $20(27.0)$ & 0.587 \\
\hline \multicolumn{5}{|l|}{ Postoperative tumor pathological features } \\
\hline Microvascular invasion & $81(54.7)$ & $30(40.5)$ & $51(68.9)$ & 0.001 \\
\hline Satellite nodules & 59 (39.9) & $18(24.3)$ & $41(55.4)$ & $<0.001$ \\
\hline Incomplete tumor encapsulation & $102(68.9)$ & $37(50.0)$ & $65(87.8)$ & $<0.001$ \\
\hline Poor tumor differentiation & $128(86.5)$ & $61(82.4)$ & $67(90.5)$ & 0.149 \\
\hline Anatomical hepatectomy & $56(37.8)$ & $28(37.8)$ & $28(37.8)$ & 1.000 \\
\hline Postoperative 30-day mortality & $0(0)$ & $0(0)$ & $0(0)$ & 1.000 \\
\hline Postoperative 30-day morbidity & $68(45.9)$ & $33(44.6)$ & $35(47.3)$ & 0.741 \\
\hline Major morbidity (Clavien-Dindo III-V) & $27(18.2)$ & $15(20.3)$ & $12(16.2)$ & 0.523 \\
\hline Minor morbidity (Clavien-Dindo I-II) & $41(27.7)$ & $18(24.3)$ & $23(31.1)$ & 0.358 \\
\hline \multicolumn{5}{|l|}{ Postoperative complications } \\
\hline Hepatic dysfunction & $15(10.1)$ & $7(9.5)$ & $8(10.8)$ & 0.785 \\
\hline Abdominal hemorrhage & $3(2.0)$ & $2(2.7)$ & $1(1.4)$ & 1.000 \\
\hline Bile leakage & $5(3.4)$ & $3(4.1)$ & $2(2.7)$ & 1.000 \\
\hline
\end{tabular}

Table 1 (continued) 
Table 1 (continued)

\begin{tabular}{|c|c|c|c|c|}
\hline Variables & Total $(n=148)$ & BS-HCC $(n=74)$ & NBS-HCC $(n=74)$ & $\mathrm{P}$ \\
\hline Organ/space infection & $9(6.1)$ & $5(6.8)$ & $4(5.4)$ & 1.000 \\
\hline Respiratory infection & $2(1.4)$ & $2(2.7)$ & $0(0)$ & 0.497 \\
\hline Pleural effusion & $45(30.4)$ & $19(25.7)$ & $26(35.1)$ & 0.211 \\
\hline Others & $16(10.8)$ & $8(10.8)$ & $8(10.8)$ & 1.000 \\
\hline
\end{tabular}

*, values are mean \pm standard deviation. AFP, alpha-fetoprotein; ASA, American Society of Anesthesiologists; BS-HCC, balloon-shaped hepatocellular carcinoma; HBV, hepatitis B virus; HCC, hepatocellular carcinoma; NBS-HCC, non-balloon-shaped hepatocellular carcinoma.

A

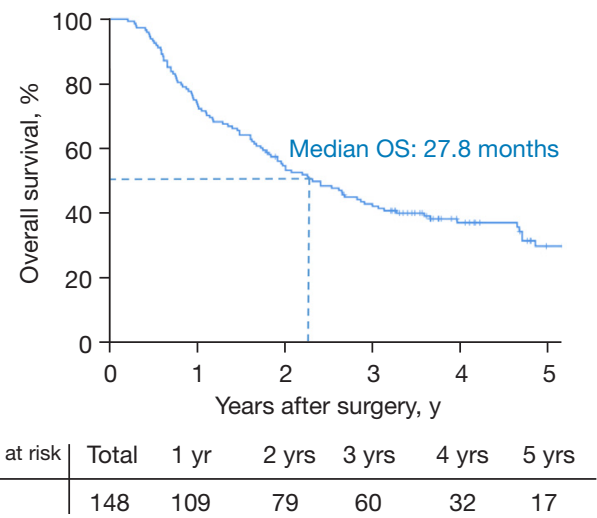

B

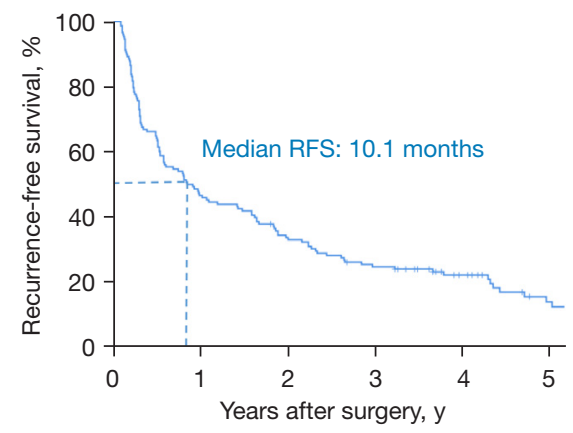

\begin{tabular}{c|cccccc} 
Patients at risk & Total & $1 \mathrm{yr}$ & $2 \mathrm{yrs}$ & $3 \mathrm{yrs}$ & $4 \mathrm{yrs}$ & $5 \mathrm{yrs}$ \\
\hline & 148 & 69 & 48 & 35 & 21 & 9
\end{tabular}

Figure 3 Curves of overall survival (OS) (A) and recurrence-free survival (RFS) (B) after liver resection for patients with a solitary huge hepatocellular carcinoma (HCC).

patients had pedunculated-growing HCCs. There were no differences in demographic and preoperative imaging characteristics between the 2 groups of patients. There were also no differences in tumor differentiation and resection margin status between the groups. The incidence of microvascular invasion $(40.5 \%$ vs. $68.9 \%, \mathrm{P}=0.001)$, satellite nodules $(24.3 \%$ vs. $55.4 \%, \mathrm{P}<0.001)$, and incomplete tumor encapsulation $(50.0 \%$ vs. $87.8 \%, \mathrm{P}<0.001)$ was different among patients with BS-HCC, as these patients had lower incidence of these adverse clinical factors than patients with NBS-HCC.

There was no postoperative 30-day death, however, the postoperative 30 -day morbidity was $45.9 \%$. There were no differences existed in likelihood of intraoperative blood transfusion, major hepatectomy, anatomical hepatectomy, postoperative 30 -day morbidity, and postoperative complications (all $\mathrm{P}>0.05$ ).

\section{Long-term outcomes}

At a median follow-up of 50.7 [95\% confidence interval (CI), 42.2-59.2] months, HCC recurrence and death occurred in $118(79.7 \%)$ and $104(70.3 \%)$ patients in the overall cohort, respectively. Median OS and RFS were 27.8 (95\% CI: 21.0-34.6) months and 10.1 (95\% CI: 5.7-14.5) months, respectively (Figure 3). Postoperative recurrence and death among patients with NBS-HCC were higher versus patients with BS-HCC $(86.5 \%$ vs. $73.0 \%, \mathrm{P}=0.041$, and $83.8 \%$ vs. $56.8 \%, \mathrm{P}<0.001$, respectively) (Table 2). The 1-, 3-, and 5 -year OS among patients with BS-HCC was $79.7 \%$, 49.3\%, and 44.8\% (median OS: 31.9 months), respectively, which were better than patients with NBS-HCC (67.6\%, $35.1 \%$, and $21.1 \%$, respectively; median OS: 21.0 months, $\mathrm{P}=0.010$, Figure $4 A$ ). The 1-, 3-, and 5-year RFS among patients with BS-HCC versus NBS-HCC were $56.8 \%$, 
Table 2 Long-term outcomes after liver resection for patients with a solitary huge hepatocellular carcinoma

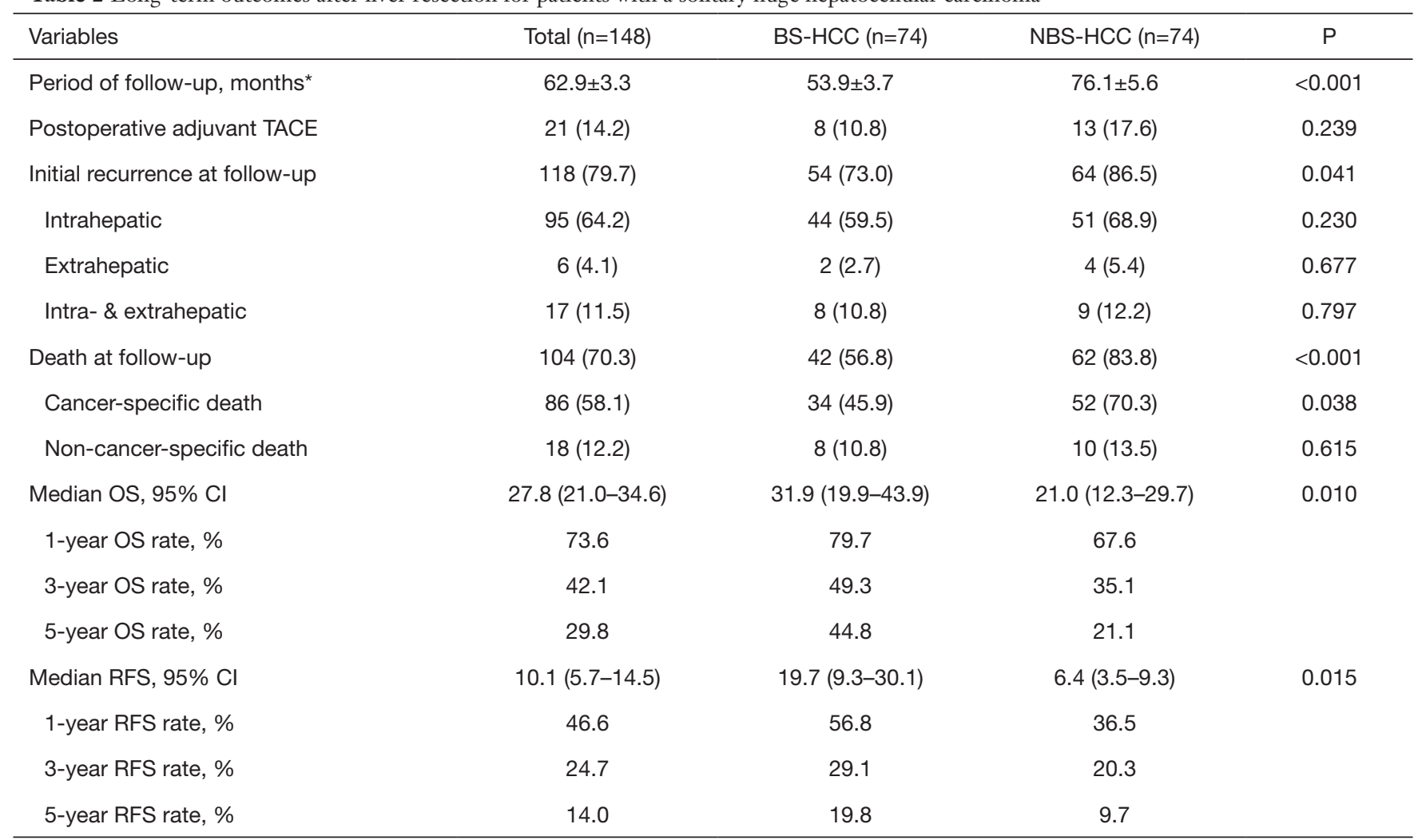

*, values are mean \pm standard deviation. BS-HCC, balloon-shaped hepatocellular carcinoma; Cl, confidence interval; HCC, hepatocellular carcinoma; NBS-HCC, non-balloon-shaped hepatocellular carcinoma; OS, overall survival; RFS, recurrence-free survival; TACE, transcatheter arterial chemoembolization.

$29.1 \%$, and $19.8 \%$ vs. $36.5 \%, 20.3 \%$ and $9.7 \%$, respectively (median RFS: 19.7 vs. 6.4 months, Figure $4 B)(\mathrm{P}=0.015)$.

Univariate and multivariate Cox-regression demonstrated several factors associated with OS and RFS following liver resection among patients with a solitary huge HCC (Table 3 and Table 4). In particular, on multivariate analyses after controlling for other clinical factors, BS-HCC remained independently associated with better OS [hazard ratio (HR) $0.592,95 \%$ CI: 0.399-0.878; $\mathrm{P}=0.009$ ] and RFS (HR 0.633, 95\% CI: 0.441-0.909; $\mathrm{P}=0.013$ ) versus NBS-HCC following curative-intent liver resection for a solitary huge HCC.

\section{Discussion}

Over the past several decades, liver resection for HCC has gradually evolved to become a safe procedure with a low perioperative mortality of less than $3 \%$ at most centers and even as low as $0 \%$ at a few large hepatic centers. However, long-term survival outcomes remain unsatisfactory mainly because of the high incidences of postoperative recurrence and metastasis (27-29). Biological characteristics based on tumor size, nodularity, encapsulation, and differentiation have prognostic significance after liver resection of solitary large/huge HCC (13-20). In our study, compared with patients who had NBS-HCC, patients with BS-HCC had better OS and RFS outcomes after curative liver resection of a solitary huge HCC. On multivariate analysis, balloonshaped tumors as identified on preoperative imaging (spherical- or ellipsoidal-shaped lesions with smooth edges) remained an independent predictive factor associated with better OS and RFS after curative liver resection for solitary huge HCC. Collectively, the data support the hypothesis that tumor morphology was an important predictor of long-term survival outcomes after curative liver resection. Thus, patients with BS-HCC should be considered for liver resection regardless of size if technically operable.

Although the results of this study found that OS 
A

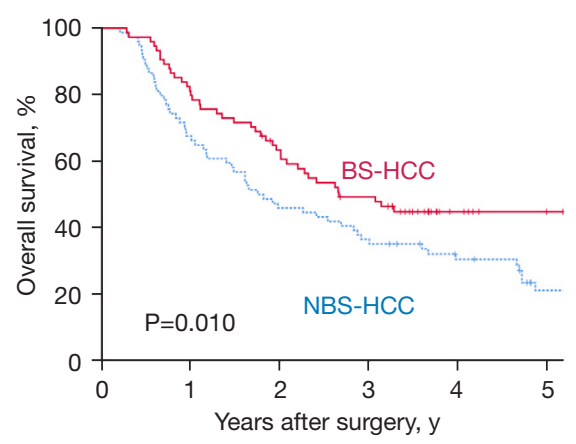

\begin{tabular}{l|cccccc} 
Patients at risk & Total & $1 \mathrm{yr}$ & 2 yrs & 3 yrs & 4 yrs & $5 y r s$ \\
\hline BS-HCC & 74 & 59 & 45 & 34 & 13 & 8 \\
NBS-HCC & 74 & 50 & 34 & 26 & 19 & 9
\end{tabular}

B

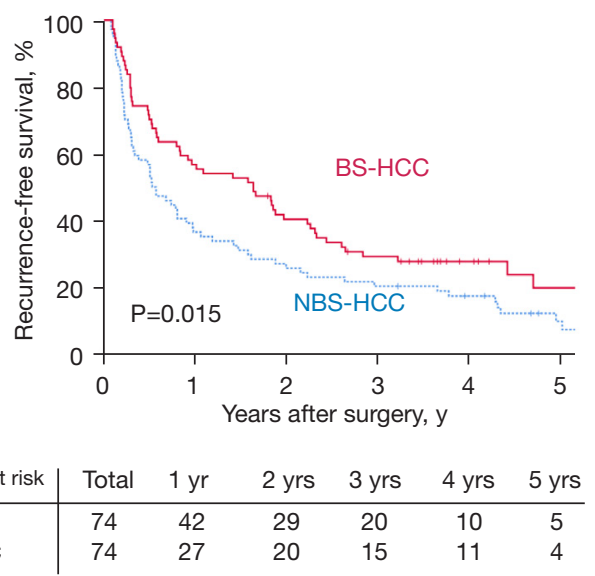

Figure 4 Comparison curves of overall survival (OS) (A) and recurrence-free survival (RFS) (B) after liver resection for a solitary huge HCC between patients with balloon-shaped hepatocellular carcinoma (BS-HCC) and non-balloon-shaped hepatocellular carcinoma (NBS-HCC).

and RFS in patients with NBS-HCC were both worse than those with BS-HCC, it did not mean that there should be any difference in the surgical indications and surgical methods between them. In our opinion, for a solitary huge HCC, if hepatectomy is carried out with curative intent and with insurance in safety, it is worth active consideration. However, in view of the significant differences in postoperative recurrence and survival outcome between patients with BS- and NBS-HCC, postoperative surveillance for recurrence should be enhanced for patients with NBS-HCC, such as shortening the surveillance interval. In addition, despite the lack of a widely recognized neoadjuvant and adjuvant treatment regimen, potentially effective treatments against recurrence are worth considering for patients with NBS-HCC, such as pre- or postoperative TACE, neoadjuvant or adjuvant systemic therapy, etc.

In several previous studies, Yang et al. defined a special type of HCC (a solitary large HCC with tumor size $>5 \mathrm{~cm}$ ) had better long-term survival outcomes after hepatectomy. Interesting, these types of tumors had a lower expression of genes associated with HCC invasion and metastatic potentials (14,30-32). In the present study, the independent impact of tumor morphology on long-term oncologic prognosis after curative resection for solitary huge HCC of $>10 \mathrm{~cm}$ was analyzed. The result demonstrated that BSHCC, a specific type of HCC with a balloon shape, had much more favorable survival outcomes with less aggressive biologic characteristics and behavior than the NBS-HCC. Table 1 demonstrates that patients with NBS-HCCs had a higher proportion of microvascular invasion, incomplete tumor encapsulation, and satellite nodules. These data supported the aggressive behavior of NBS-HCC with this tumor morphology. As a tumor capsule is composed of thick collagen fibers and vascular structures, complete tumor encapsulation has been recognized as a protective barrier in confining the tumor and preventing spread of tumor cells $(33,34)$. Encapsulated HCCs have also been correlated with lower incidence of aggressive tumor characteristics like microvascular invasion and satellite nodules, and better OS and RFS outcomes after liver resection $(18,33)$.

In this study, the classification of balloon or non-balloon shape was based on preoperative CT or MRI imaging within 1 month before surgery. Of note, HCCs with different shapes are not hard to distinguish on imaging. In particular, in the current study, a radiologist and two surgeons who participated in the imaging evaluations were able to reach agreement in over $90 \%$ of cases on initial assessment. The definition of "balloon-shaped" was determined based on spherical or ellipsoidal shape, as well as a low- or high-density shadow around the intact tumor capsule. In the entire cohort of 377 patients before PSM, unanimous judgement on identification of BS or NBS was reached on imaging in almost all the patients $(\mathrm{N}=358$, $95.0 \%$ ), and discrepancies on the judgement of low- or high-density peripheral rim around the lesion existed only in 19 cases $(5.0 \%)$. However, after discussion, a consensus was easily and completely reached for these 19 cases. To test the impact of these 19 cases, further sensitivity analysis was made, and the conclusions did not change. In the BSHCC group, the proportion of specimens with complete tumor encapsulation on postoperative histopathological 
Table 3 Univariate and multivariate Cox-regression analysis predicting overall survival after liver resection of patients with a solitary huge hepatocellular carcinoma

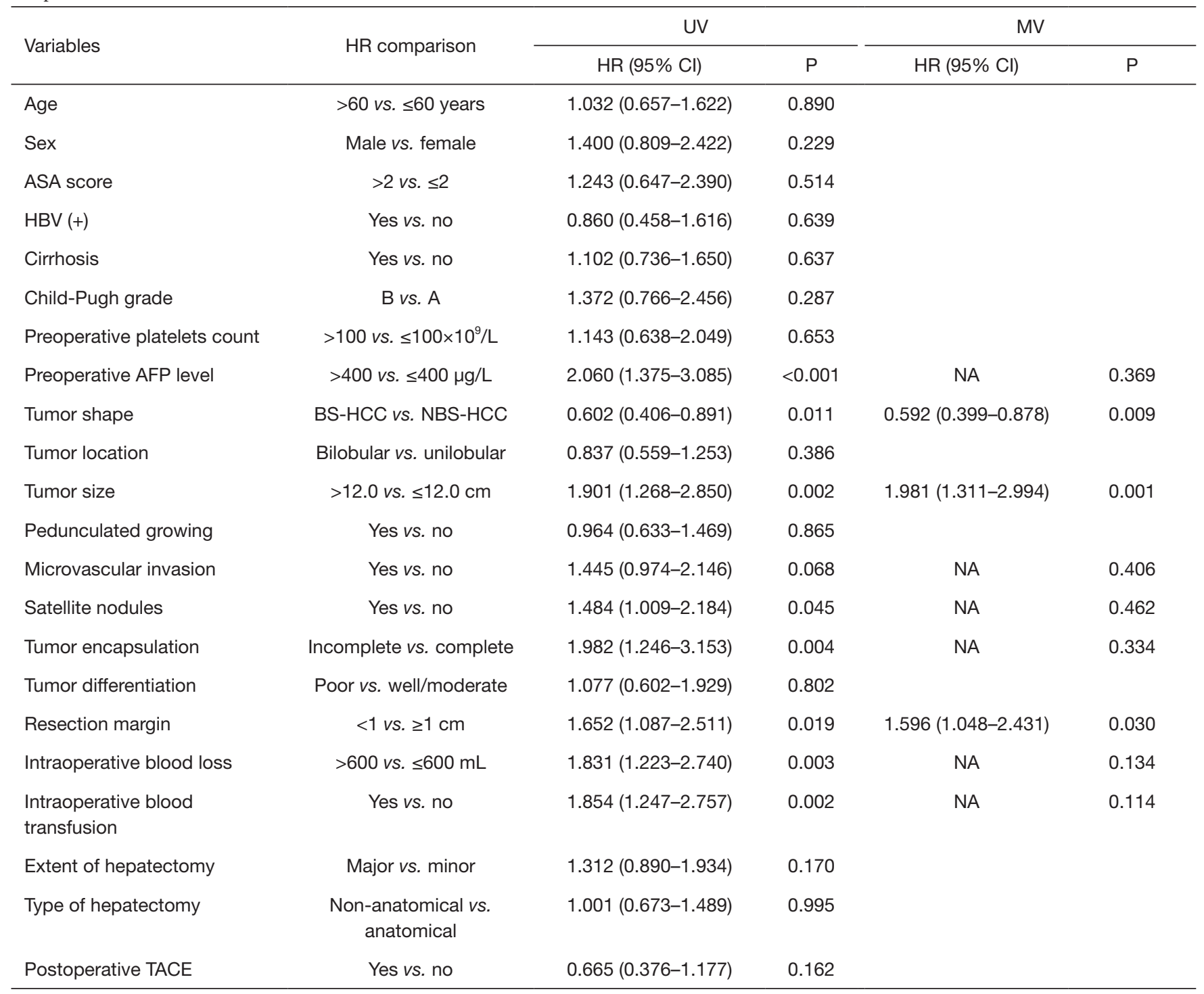

*, variables with $\mathrm{P}<0.1$ in univariate analysis were subjected to multivariate Cox-regression model using forward stepwise variable selection. AFP, alpha-fetoprotein; ASA, American Society of Anesthesiologists; BS-HCC, balloon-shaped hepatocellular carcinoma; CI, confidence interval; HR, hazard ratio; MV, multivariate; NA, not available; UV, univariate; NBS-HCC, non-balloon-shaped hepatocellular carcinoma; TACE, transcatheter arterial chemoembolization.

examination was $50 \%$, which was much higher than the NBS-HCC group (only $12.2 \%, \mathrm{P}<0.001$ ). On univariate analysis, patients with complete tumor encapsulation had better OS and RFS than patients with incomplete tumor encapsulation $(\mathrm{P}=0.004$ and 0.015$)$. Although whether tumor encapsulation is complete or incomplete can only be confirmed by postoperative histopathological examination of resected specimens, preoperative imaging findings can be used in its prediction.

Interestingly, a large proportion of patients with resectable solitary huge HCCs, including the balloon or non-balloon types, had pedunculated growth tumors (29.1\%, 43/148) (i.e., half of the tumors protruded from the surfaces of the livers, it is common for small HCCs to have a capsule). When HCC tumors grow rapidly, there may be increasingly more pressure exerted by the surrounding liver 
Table 4 Univariate and multivariate Cox-regression analysis predicting recurrence-free survival after liver resection of patients with a solitary huge hepatocellular carcinoma

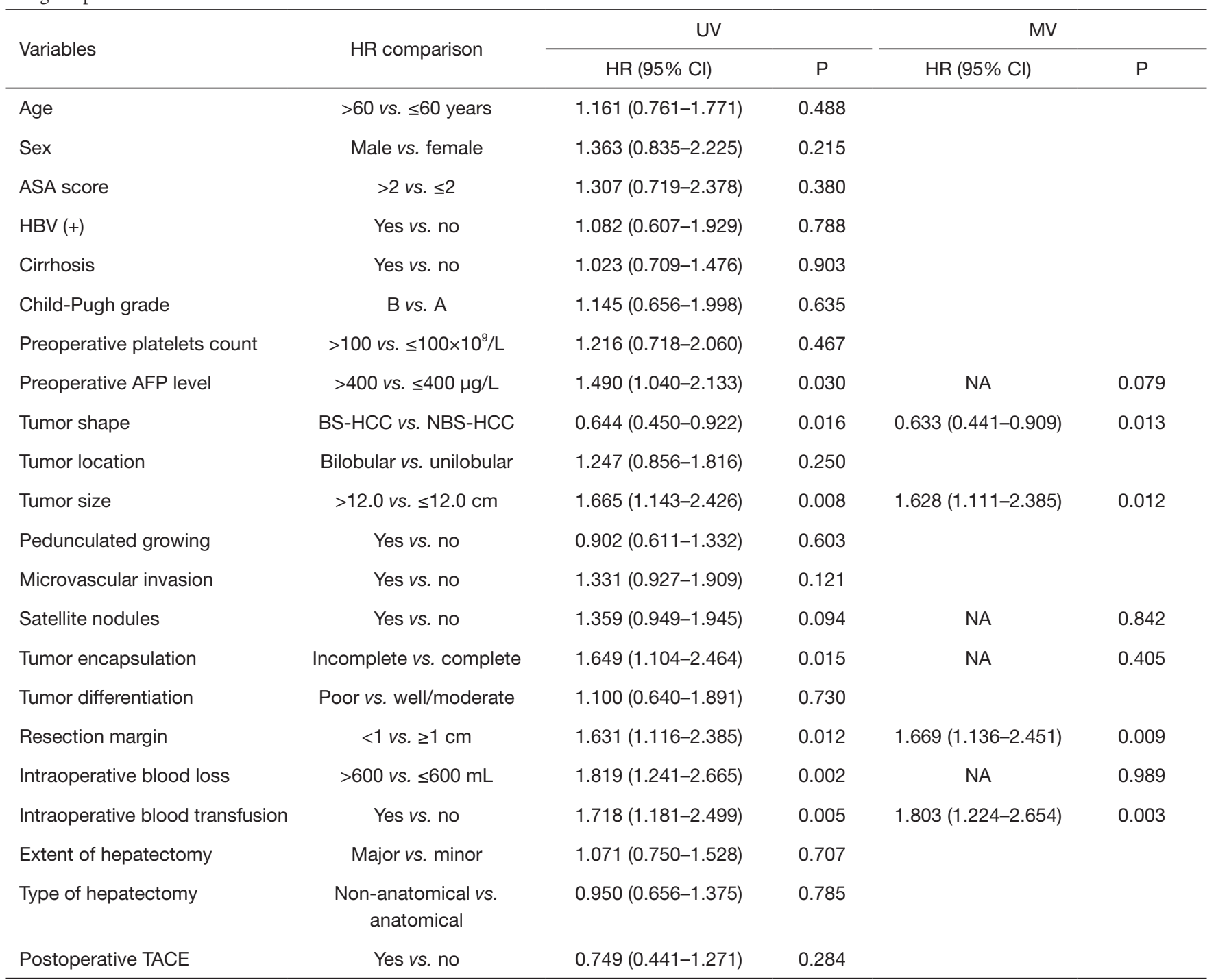

*, variables with $\mathrm{P}<0.1$ in univariate analysis were subjected to multivariate Cox-regression model using forward stepwise variable selection. AFP, Alpha-fetoprotein; ASA, American Society of Anesthesiologists; BS-HCC, Balloon-shaped hepatocellular carcinoma; CI, confidence interval; HR, hazard ratio; MV, multivariate; NA, not available; UV, univariate; NBS-HCC, non-balloon-shaped hepatocellular carcinoma; TACE, transcatheter arterial chemoembolization.

parenchyma on the HCC if it is centrally located in the liver versus a peripherally located tumor. Thus, there is a higher tendency for a centrally located tumor to break through the constraints of a capsule or pseudo-capsule to obtain the required growth space than a peripherally located HCC. Once breaking through the capsule, the tumor may grow uncontrollably and become irregular in shape. As a result, the chances of microsatellite lesions and the development of multiple secondary lesions can greatly increase. In pedunculated HCCs that often arise in peripherally located areas, these lesions are technically easier to resect with lower incidence of postoperative recurrence, and better long-term survival $(35,36)$. Of note, the chance of tumor rupture for pedunculated HCCs is higher, as one study reported that among 143 patients with ruptured HCC reported $35 \%$ were pedunculated in nature (37).

All patients in our cohort underwent open hepatectomy, and none of them underwent minimally invasive hepatectomy (laparoscopic or robotic). Till now, minimally invasive hepatectomy for huge HCC is still controversial, 
although some studies have reported that huge HCC was not a contraindication for minimally invasive hepatectomy, and the perioperative morbidity and mortality rates were comparable to those of open hepatectomy $(38,39)$. In our experience, laparoscopic hepatectomy for huge HCCs is full of challenges and difficulties, and the probability of intraoperative tumor rupture, especially if carried out by surgeons who are not very experienced in laparoscopic surgery. More prospective observational studies and even randomized clinical trials are needed to further determine the safety and efficacy of minimally invasive hepatectomy to compare with open hepatectomy for huge HCC. Currently, radiomics and machine learning are hot research topics, which have shown great application value in diagnosis, prognosis, treatment selection and monitoring of response to treatment for malignant tumors, including HCC (40-42). We have already stated on research on prognostic evaluation for solitary huge HCC by using these technologies.

Several limitations need to be considered when interpreting data from the current study. As a retrospective study, there may be inherent residual biases despite PSM. The vast majority (nearly 90\%) of patients also had HBVrelated HCC. As such, external validation in a Western cohort of patients in which HCV and alcoholism are the main etiological causes of HCC is needed. In addition, the current multi-institutional study did not allow for standardization of protocols related to liver resection technique. Furthermore, all operations were performed by experienced surgeons at large specialized surgical centers in China who had experience in managing HCC patients. In turn, the results may not be generalizable in small centers or to less experienced surgeons.

In conclusion, the data demonstrated that liver resection can safely and effectively be performed in patients with a solitary huge HCC $>10 \mathrm{~cm}$, with reasonably good median OS and RFS rates. Preoperative imaging categorization of tumor morphology was an important prognostic factor relative to long-term OS and RFS outcomes following curative-intent resection of HCC.

\section{Acknowledgments}

Funding: This work was supported by National Natural Science Foundation of China (No. 81972726).

\section{Footnote}

Reporting Checklist: The authors have completed the
STROBE reporting checklist. Available at https://hbsn. amegroups.com/article/view/10.21037/hbsn-21-423/rc

Data Sharing Statement: Available at https://dx.doi. org/10.21037/hbsn-21-423/dss

Conflicts of Interest: All authors have completed the ICMJE uniform disclosure form (available at https://dx.doi. org/10.21037/hbsn-21-423/coif). TMP and WYL serve as the unpaid editorial board members of Hepatobiliary Surgery and Nutrition. The other authors have no conflicts of interest to declare.

Ethical Statement: The authors are accountable for all aspects of the work in ensuring that questions related to the accuracy or integrity of any part of the work are appropriately investigated and resolved. The study was performed in accordance with the Declaration of Helsinki and the Ethical Guidelines for Clinical Studies for the enrolled centers. Informed consent was waived by the Institutional Review Board of EHBH (No. EHBHKY2019-K-005). Consent for the relevant procedures and the use of data for research purposes were obtained from all the patients before treatment.

Open Access Statement: This is an Open Access article distributed in accordance with the Creative Commons Attribution-NonCommercial-NoDerivs 4.0 International License (CC BY-NC-ND 4.0), which permits the noncommercial replication and distribution of the article with the strict proviso that no changes or edits are made and the original work is properly cited (including links to both the formal publication through the relevant DOI and the license). See: https://creativecommons.org/licenses/by-nc-nd/4.0/.

\section{References}

1. Llovet JM, Kelley RK, Villanueva A, et al. Hepatocellular carcinoma. Nat Rev Dis Primers 2021;7:6.

2. Vibert E, Schwartz M, Olthoff KM. Advances in resection and transplantation for hepatocellular carcinoma. J Hepatol 2020;72:262-76.

3. Heimbach JK, Kulik LM, Finn RS, et al. AASLD guidelines for the treatment of hepatocellular carcinoma. Hepatology 2018;67:358-80.

4. European Association for the Study of the Liver. Electronic address: easloffice@easloffice.eu; European Association for the Study of the Liver. EASL Clinical 
Practice Guidelines: Management of hepatocellular carcinoma. J Hepatol 2018;69:182-236.

5. Marrero JA, Kulik LM, Sirlin CB, et al. Diagnosis, Staging, and Management of Hepatocellular Carcinoma: 2018 Practice Guidance by the American Association for the Study of Liver Diseases. Hepatology 2018;68:723-50.

6. Wang L, Liu Z, Liu X, et al. The hepatectomy efficacy of huge hepatocellular carcinoma and its risk factors: A meta analysis. Medicine (Baltimore) 2017;96:e9226.

7. Wakayama K, Kamiyama T, Yokoo H, et al. Huge hepatocellular carcinoma greater than $10 \mathrm{~cm}$ in diameter worsens prognosis by causing distant recurrence after curative resection. J Surg Oncol 2017;115:324-9.

8. Lim C, Compagnon P, Sebagh M, et al. Hepatectomy for hepatocellular carcinoma larger than $10 \mathrm{~cm}$ : preoperative risk stratification to prevent futile surgery. HPB (Oxford) 2015;17:611-23.

9. Yang L, Xu J, Ou D, et al. Hepatectomy for huge hepatocellular carcinoma: single institute's experience. World J Surg 2013;37:2189-96.

10. Torzilli G, Belghiti J, Kokudo N, et al. A snapshot of the effective indications and results of surgery for hepatocellular carcinoma in tertiary referral centers: is it adherent to the EASL/AASLD recommendations?: an observational study of the HCC East-West study group. Ann Surg 2013;257:929-37.

11. Pinna AD, Yang T, Mazzaferro V, et al. Liver Transplantation and Hepatic Resection can Achieve Cure for Hepatocellular Carcinoma. Ann Surg 2018;268:868-75.

12. Zaydfudim VM, Vachharajani N, Klintmalm GB, et al. Liver Resection and Transplantation for Patients With Hepatocellular Carcinoma Beyond Milan Criteria. Ann Surg 2016;264:650-8.

13. Stevens CL, Awad A, Abbas SM, et al. Systematic review and meta-analysis of hepatic resection versus transarterial chemoembolization for solitary large hepatocellular carcinoma. HPB (Oxford) 2017;19:653-8.

14. Yang LY, Fang F, Ou DP, et al. Solitary large hepatocellular carcinoma: a specific subtype of hepatocellular carcinoma with good outcome after hepatic resection. Ann Surg 2009;249:118-23.

15. Liu PH, Su CW, Hsu CY, et al. Solitary Large Hepatocellular Carcinoma: Staging and Treatment Strategy. PLoS One 2016;11:e0155588.

16. Zhou L, Rui JA, Wang SB, et al. Prognostic factors of solitary large hepatocellular carcinoma: the importance of differentiation grade. Eur J Surg Oncol 2011;37:521-5.

17. Choi GH, Han DH, Kim DH, et al. Outcome after curative resection for a huge ( $>$ or $=10 \mathrm{~cm}$ ) hepatocellular carcinoma and prognostic significance of gross tumor classification. Am J Surg 2009;198:693-701.

18. Wu TH, Yu MC, Chen TC, et al. Encapsulation is a significant prognostic factor for better outcome in large hepatocellular carcinoma. J Surg Oncol 2012;105:85-90.

19. Zhong JH, Pan LH, Wang YY, et al. Optimizing stage of single large hepatocellular carcinoma: A study with subgroup analysis by tumor diameter. Medicine (Baltimore) 2017;96:e6608.

20. Lim C, Mise Y, Sakamoto Y, et al. Above $5 \mathrm{~cm}$, size does not matter anymore in patients with hepatocellular carcinoma. World J Surg 2014;38:2910-8.

21. Pawlik TM, Delman KA, Vauthey JN, et al. Tumor size predicts vascular invasion and histologic grade: Implications for selection of surgical treatment for hepatocellular carcinoma. Liver Transpl 2005;11:1086-92.

22. Carr BI, Guerra V, Donghia R, et al. Trends in Tumor Indices in Relation to Increased Hepatocellular Carcinoma Size: Evidence for Tumor Evolution as a Function of Growth. J Gastrointest Cancer 2020;51:1215-9.

23. Yang LY, Wang W, Peng JX, et al. Differentially expressed genes between solitary large hepatocellular carcinoma and nodular hepatocellular carcinoma. World J Gastroenterol 2004;10:3569-73.

24. Wang W, Yang LY, Huang GW, et al. Genomic analysis reveals $\mathrm{RhoC}$ as a potential marker in hepatocellular carcinoma with poor prognosis. Br J Cancer 2004;90:2349-55.

25. Strasberg SM, Phillips C. Use and dissemination of the brisbane 2000 nomenclature of liver anatomy and resections. Ann Surg 2013;257:377-82.

26. EDMONDSON HA, STEINER PE. Primary carcinoma of the liver: a study of 100 cases among 48,900 necropsies. Cancer 1954;7:462-503.

27. Chang YJ, Chung KP, Chang YJ, et al. Long-term survival of patients undergoing liver resection for very large hepatocellular carcinomas. Br J Surg 2016;103:1513-20.

28. Levi Sandri GB, Spoletini G, Vennarecci G, et al. Laparoscopic liver resection for large HCC: short- and long-term outcomes in relation to tumor size. Surg Endosc 2018;32:4772-9.

29. Zhang H, Yuan SX, Dai SY, et al. Tumor size does not independently affect long-term survival after curative resection of solitary hepatocellular carcinoma without macroscopic vascular invasion. World J Surg 2014;38:947-57.

30. Yang LY, Tao YM, Ou DP, et al. Increased expression 
of Wiskott-Aldrich syndrome protein family verprolinhomologous protein 2 correlated with poor prognosis of hepatocellular carcinoma. Clin Cancer Res 2006;12:5673-9.

31. Chang ZG, Yang LY, Wang W, et al. Determination of high mobility group A1 (HMGA1) expression in hepatocellular carcinoma: a potential prognostic marker. Dig Dis Sci 2005;50:1764-70.

32. Huang GW, Yang LY, Lu WQ. Expression of hypoxiainducible factor 1alpha and vascular endothelial growth factor in hepatocellular carcinoma: Impact on neovascularization and survival. World J Gastroenterol 2005;11:1705-8.

33. Lim JH, Choi D, Park CK, et al. Encapsulated hepatocellular carcinoma: CT-pathologic correlations. Eur Radiol 2006;16:2326-33.

34. Iguchi T, Aishima S, Sanefuji K, et al. Both fibrous capsule formation and extracapsular penetration are powerful predictors of poor survival in human hepatocellular carcinoma: a histological assessment of 365 patients in Japan. Ann Surg Oncol 2009;16:2539-46.

35. Nishizaki T, Matsumata T, Adachi E, et al. Pedunculated hepatocellular carcinoma and surgical treatment. Br J Cancer 1993;67:115-8.

36. Yeh CN, Lee WC, Jeng LB, et al. Pedunculated hepatocellular carcinoma: clinicopathologic study of 18

Cite this article as: $\mathrm{Xu} \mathrm{XF,} \mathrm{Wu} \mathrm{H,} \mathrm{Li} \mathrm{JD,} \mathrm{Yao} \mathrm{LQ,} \mathrm{Huang}$ B, Diao YK, Chen TH, Gu WM, Chen Z, Li J, Zhang YM, Wang H, Liang YJ, Zhou YH, Li C, Wang MD, Zhang CW, Pawlik TM, Lau WY, Shen F, Yang T. Association of tumor morphology with long-term prognosis after liver resection for patients with a solitary huge hepatocellular carcinoma-a multicenter propensity score matching analysis. HepatoBiliary Surg Nutr 2022. doi: 10.21037/hbsn-21-423 surgically resected cases. World J Surg 2002;26:1133-8.

37. Yang T, Sun YF, Zhang J, et al. Partial hepatectomy for ruptured hepatocellular carcinoma. Br J Surg 2013;100:1071-9.

38. Gumbs AA, Leventhal A, Hoffman JP. Video: laparoscopic right hepatectomy and partial resection of the diaphragm for liver metastases. Surg Endosc 2011;25:3441-3.

39. Cheung TT, Wang X, Efanov M, et al. Minimally invasive liver resection for huge $(\geq 10 \mathrm{~cm})$ tumors: an international multicenter matched cohort study with regression discontinuity analyses. Hepatobiliary Surg Nutr 2021;10:587-97.

40. Xu X, Zhang HL, Liu QP, et al. Radiomic analysis of contrast-enhanced CT predicts microvascular invasion and outcome in hepatocellular carcinoma. J Hepatol 2019;70:1133-44.

41. Ji GW, Zhu FP, Xu Q, et al. Machine-learning analysis of contrast-enhanced CT radiomics predicts recurrence of hepatocellular carcinoma after resection: A multiinstitutional study. EBioMedicine 2019;50:156-65.

42. Feng B, Ma XH, Wang S, et al. Application of artificial intelligence in preoperative imaging of hepatocellular carcinoma: Current status and future perspectives. World J Gastroenterol 2021;27:5341-50. 

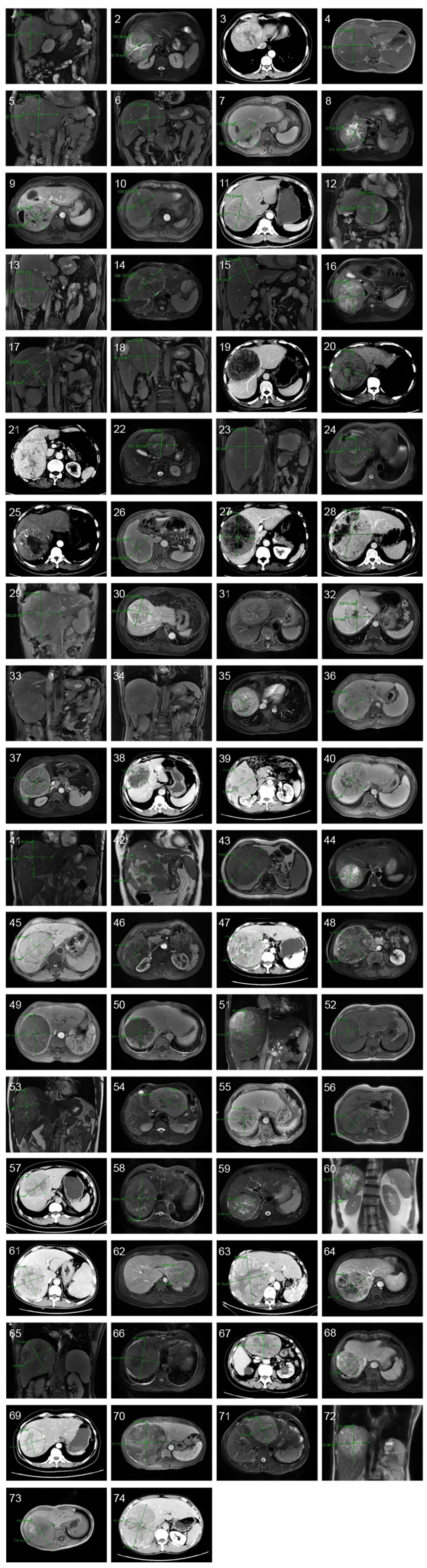


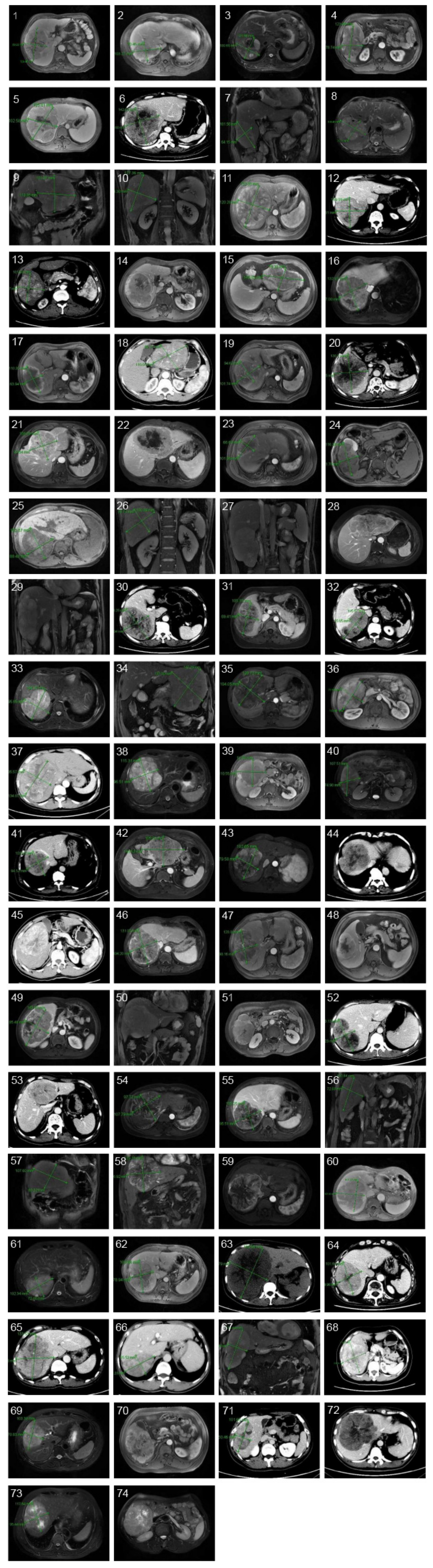

Figure S2 Non-balloon-shaped Solitary Huge HCC. 